\section{Gemeindebeteiligung als Schlüssel für regionale Akzeptanz}

\author{
Das Beispiel des Stollenspeicher- \\ Wasserkraftwerkes Stanzertal
}

\begin{abstract}
von Wolfgang Widmann, Manfred Roner und Robert Weidner, Infra Project Development GmbH, Innsbruck, Österreich
\end{abstract}

\begin{abstract}
Durch eine frühe Einbindung der Gemeinden und betroffener Bürger in die Projektentwicklung sowie deren Beteiligung an der Projektgesellschaft, kann die bei der Umsetzung von Infrastrukturprojekten häufig auftretende ablehnende Haltung in der Bevölkerung überwunden werden. Voraussetzung dafür ist, dass es sich um ein ökologisch verträgliches und zugleich wirtschaftliches Projekt handelt. Schlüssel für das Erreichen der regionalen Akzeptanz ist der Aufbau eines Vertrauensverhältnisses unter den Projektpartnern. Dies gelingt durch Transparenz, ergebnisoffene Diskussionen, Beteiligung an Entscheidungen und glaubwürdiges Bemühen, den gemeinsamen Projekterfolg über die Eigeninteressen zu stellen.
\end{abstract}

With the early engagement of communities and stakeholders in the development of the project as well as their engagement in the project association the often reluctant positions of the public regarding the realization of infrastructure projects can be overcome. For this it is a requirement that the project is ecologically compliant and at the same time economically beneficial. Key to reaching regional acceptance is building mutual trust among the project partners. This is possible through transparency, open discussions, participation regarding decisions and a creditable effort to put the collected project success before self-interests.

\section{Ausgangslage}

In Mitteleuropa hat sich in den letzten Jahrzehnten großer Wohlstand entwickelt und die gesellschaftlichen Rahmenbedingungen zur Umsetzung von Infrastrukturprojekten haben sich geändert. Selbstbewusste Bürger, oftmals unterstützt durch lokale und überregionale Medien, suchen Wege, subjektive Interessen und Befürchtungen $\mathrm{zu}$ artikulieren und durchzusetzen. Zusätzlich werden häufig Plattformen und soziale Netzwerke mit hoher gesellschaftlicher Reichweite genutzt, worauf Unternehmen zumeist Schwierigkeiten haben, adäquat zu reagieren.

Diese Entwicklung bringt es mit sich, dass Infrastrukturprojekte von der ersten Idee an kritisch begleitet werden können. Die technische Machbarkeit ist dabei selten Gegenstand der Diskussion, sie wird vorausgesetzt. Die Umsetzung ökologischer Maßnahmen auf Grundlage gesetzlicher Vorgaben und behördlicher Auflagen wird schon eher misstrauisch betrachtet. Und selbst die Einhaltung aller Vorgaben und Auflagen stellt keine Garantie für eine allgemeine Zustimmung zum Projekt dar.

Als Projektgesellschaft, die Infrastrukturprojekte begleitet und durchführt, beobachten wir häufig das Floriani-Prinzip ${ }^{1}$ oder die als NIMBY$^{2}$-Mentalität bekannte Haltung. Wenn die Mehrheit der Tiroler Bevölkerung den Ausbau der Wasserkraft prinzipiell gut heißt, allerdings diese Zustimmung rapide sinkt, sobald die eigene Umgebung davon betroffen ist, dann ist das ein gutes Beispiel für den Mangel an regionaler Akzeptanz von Infrastrukturprojekten, obwohl deren generelle Notwendigkeit eingesehen wird (Bliem 2013). Deshalb muss bei der Planung und Realisierung eines Infrastrukturprojektes diese Realität als solche erkannt werden und glaubwürdige Maßnahmen zur Überwindung möglicher Vorbehalte in der Region gesetzt werden.

Im vorliegenden Beitrag wird ein mit Stollenspeicher ausgestattetes Wasserkraftwerk in Tirol beschrieben, bei dem es der Projektenwicklungsfirma und den betroffenen Gemeinden gelungen ist, in der Bevölkerung regionale Akzeptanz des Projektes zu erreichen. Zudem werden die gewählte Vorgangsweise und die dabei gewonnenen Erfahrungen geschildert.

\section{Projektbeschreibung}

Das Wasserkraftwerk Stanzertal ist ein Ausleitungskraftwerk an der Rosanna in Westtirol mit einer Engpassleistung von 13,5 MW, einer Ausbauwassermenge von $12 \mathrm{~m}^{3} / \mathrm{s}$ und einem Regeljahresarbeitsvermögen von 52,2 GWh. Die Hauptbauteile sind: 
- Wehr mit Fischbauchklappe und Fischaufstieg

- Seitenentnahme mit dreikammrigem Entsander

- 4,8 km langer Speicherstollen (Ausbruchsdurchmesser $3,8 \mathrm{~m})$

- $100 \mathrm{~m}$ stahlgepanzerter Lotschacht mit 2,2 m Innendurchmesser

- 500 m lange Druckrohrleitung DN 2000, großteils in einem Tunnel verlegt

- Krafthaus mit drei Maschinensätzen (Peltonturbinen; Nettofallhöhe 135 m)

- Energieableitung über ein $25 \mathrm{kV}$ Kabel

- Pumpwerk mit Rohrleitung zum Einleiten des geklärten Abwassers aus der Region in den Triebwasserweg

- Ausgleichsfläche mit Biotopen und Verbesserungen des Fischlebensraumes an der Rosanna

Mit Gesamtkosten von 57,6 Mio. Euro auf Preisbasis 2015 und inklusive aller Finanzierungsund Nebenkosten und Entschädigungen ist das Kraftwerk auch wirtschaftlich günstig.

\subsection{Technische Beschreibung}

Eine technische Besonderheit stellt der Speicherstollen dar, der es ermöglicht - immer wenn der Zufluss kleiner als die Ausbauwassermenge ist - die Erzeugung dem Bedarf anzupassen und somit einen wertvollen Beitrag zur Residuallastabdeckung zu leisten.

Bei der Stollenspeicherung wird der Triebwasserstollen eines Ausleitungskraftwerkes so konzipiert, dass er sowohl zum Transport als auch zur Speicherung des Triebwassers genutzt werden kann. Günstig ist ein langer Triebwasserstollen mit großem Durchmesser und geringem Gefälle. Abbildung 1 gibt einen schematischen Längsschnitt der Kraftwerksanlage wieder, in welchem der Speicherstollen ersichtlich ist.

Das zur Verfügung stehende Speichervolumen ist neben der baulichen Dimensionierung direkt vom nutzbaren Zufluss abhängig und erreicht sein Maximum in der Niederwasserperiode. Mit steigendem Abfluss sinkt das nutzbare Speichervolumen, da ein größerer Teil des Stollenquerschnitts für den Wassertransport benötigt wird. Da der spezifische Energieinhalt eines Kubikmeters Speichervolumen mit der Fallhöhe des Kraftwerks steigt, eignet sich dieses Konzept besonders für Mittel- und Hochdruckanlagen im Tagesspeicherbetrieb.

Die gesamte Anlage muss technisch so umgesetzt werden, dass die mit der Speicherbewirtschaftung verbundenen kurzfristigen Wasserspiegellagenänderungen sowie der Wechsel vom Abfluss bei Voll- und Teilfüllung im Triebwasserstollen zu keinen negativen Auswirkungen auf die verschiedenen Kraftwerkskomponenten führen, sodass eine aktive Speicherbewirtschaftung mit raschen Lastwechseln und häufigen Speicherzyklen umgesetzt werden kann.

Mit dem nutzbaren Speichervolumen des WKW Stanzertal von rund $50.000 \mathrm{~m}^{3}$ können etwa $15 \%$ der mittleren Jahresproduktion tageszeitlich verlagert werden.

\subsection{Vermarktungsmöglichkeiten}

Angesichts der Entwicklung des Kraftwerkportfolios mit steigendem Anteil an Wind- und Photovoltaikproduktion im deutsch-österreichischen Marktgebiet steigt der Bedarf an regelbarer Stromerzeugung.

Einerseits spiegelt sich der Einfluss der PVProduktion in einem stark gesunkenen Preisni- 
veau während den Mittagsstunden wider, sodass es derzeit bei den Preisen zu einer Morgen- und Abendspitze kommt. Andererseits ist das gesamte Preisniveau stark gesunken, was die möglichen Erträge von Laufwasserkraftwerken schmälert.

Mittels Stollenspeicher kann das WKW Stanzertal die oben beschriebenen Ertragseinbußen zumindest teilweise kompensieren. Beispielsweise wird durch die flexible Betriebsführung für die Stromvermarktung im Jahr 2015 ein Mehrertrag von $20 \%$ gegenüber einem Betrieb als reines Laufwasserkraftwerk erzielt.

Zusätzlich ermöglicht der Stollenspeicher die Verfolgung wesentlich kurzfristigerer Vermarktungsstrategien, um das Wertsteigerungspotenzial bestmöglich auszuschöpfen:

- Day-Ahead-Markt

Am Day-Ahead-Markt wird Strom mittels täglicher Auktion für den Folgetag gehandelt. Die Vermarktungsmöglichkeiten werden dabei wie folgt unterteilt:

- stündliche Einsatzoptimierung

Bei der stündlichen Betriebsoptimierung findet eine Produktionsverlagerung von den Nacht- und Mittagsstunden zu den täglichen Bedarfsspitzen statt.

Dies hätte am Day-Ahead-Markt der Leipziger Strombörse im Jahre 2012 zu einem Mehrertrag von $6 \%$ gegenüber einem reinen Laufkraftwerk geführt (e3 consult GmbH 2013).

- viertelstündliche Einsatzoptimierung Durch den 15-Minuten-Handel können innerhalb einer Stunde hohe Abweichungen zwischen den einzelnen Viertelstundenprodukten genutzt werden um den Speicher bestmöglich zu bewirtschaften. Im Gegensatz zur stündlichen Einsatzoptimierung stellt die Speichergröße bei der viertelstündlichen Optimierung durch häufigere Speicherzyklen einen wesentlich geringeren Einflussfaktor dar.

\section{- Intraday-Markt}

Der Intraday-Markt erlaubt dem Kraftwerksbetreiber, je nach Lieferzone bis zu 45 (Amprion, TenneT, 50Hertz, TransnetBW) bzw. 75 Minuten (APG) vor physikalischer Lieferung die Stromproduktion anzupassen um Prognoseabweichungen kurzfristig auszuglei- chen. Dem Intraday-Markt wird aufgrund des fortschreitenden Ausbaus erneuerbarer Energien und der damit verbundenen steigenden volatilen Einspeisung immer mehr Bedeutung prognostiziert (Süßenbacher et al. 2015).

- Bereitstellung von Regelenergie

Im Bereich der Systemdienstleistungen sind Laufkraftwerke in der Lage, durch Reduktion der Erzeugung negative Regelenergie bereit zu stellen. Je nach Anforderung und/ oder Eingliederung in einem virtuellen Kraftwerkspool kann das WKW Stanzertal positive sowie negative Regelenergie bereitstellen.

- Kurzfristige Over-The-Counter-Geschäfte

- Ausregelung von Leistungsunausgeglichenheiten innerhalb einer Bilanzgruppe zur Vermeidung von Ausgleichsenergiekosten

Es wird erwartet, dass flexible Energieerzeugung aus ressourcenschonenden, erneuerbaren Energiequellen in Zukunft v. a. zur Residuallastabdeckung weiter an Bedeutung zunimmt und das Upside-Potenzial bzw. der Mehrertrag steigt.

\section{Gemeindebeteiligung}

\subsection{Regionale Akzeptanz durch Einbindung der Standortgemeinden}

Eine der besten Möglichkeiten zur Erhöhung der regionalen Akzeptanz für ein Infrastrukturprojekt wie das beschriebene Wasserkraftwerk Stanzertal liegt darin, die Projektgestaltung und die Projektentwicklung in partnerschaftlicher Form gemeinsam mit der oder den betroffenen Gemeinden als Vertreter der in der Region lebenden Bürger vorzunehmen (Götz et al. 2011). Das beginnt idealerweise damit, dass die Grundidee vom Projektentwickler und von den Gemeinden ähnlich gesehen wird. Bereits die ersten Umsetzungskonzepte - und vor allem diese - müssen mit der Gemeinde besprochen und prinzipiell geklärt werden. Während der Projektentwickler für die technisch, wirtschaftlich und ökologisch optimale Gestaltung des Projektes verantwortlich ist, sorgt die Gemeinde dafür, dass auch nicht-technische und nicht-monetäre Kriterien aus dem regionalen Umfeld (Infrastruktur, Regionalentwicklung, standortspezifische Besonderheiten) berücksichtigt werden. Dieses Zusammenspiel aller Beteiligten führt dazu, dass: 
- das Projekt in das regionale Umfeld optimal eingefügt wird,

- der Gemeinde die relevanten Eckpunkte des Projektes vermittelt werden,

- eine Vertrauensbasis zwischen beiden Partnern aufgebaut wird.

Nach der gemeinsamen Klärung der wichtigsten Eckpunkte muss das Projektkonzept auch den Bürgern und den Projektbetroffenen vorgestellt und mit ihnen diskutiert werden. Anschließend müssen die Entscheidungen für die weitere Vorgehensweise weitgehend gemeinsam getroffen werden. Wie die Kommunikation im Detail ablaufen soll, muss von Fall zu Fall geplant werden. Es braucht in angemessener Form und in ausreichendem Umfang Informationen für die Gemeinderäte, die Betroffenen und die interessierte Bevölkerung. Kritik und Skepsis müssen aufgenommen und sorgfältig beachtet werden, weil dabei meistens bisher unbeachtete und oft wichtige Details angesprochen werden. Zugleich ist es wichtig, den Gestaltungsspielraum der Gemeinden, Betroffenen und Bürger $\mathrm{zu}$ definieren, um keine falschen und unerfüllbaren Hoffnungen zu wecken, die dann unweigerlich zu Enttäuschung und Misstrauen führen würden.

\subsection{Aufteilung der Aufgaben und Risiken}

Das Ziel der gemeinsamen Projektentwicklung ist es, die jeweiligen Stärken des Partners für das Gelingen des Projektes zu nutzen. Die Stärke der Gemeinden liegt in der Kompetenz, politisch einschätzen zu können, was in der Region möglich und sinnvoll ist, um damit die regionale Akzeptanz zu erhöhen. Der Projektentwickler bringt seine Kompetenz in die technisch, wirtschaftlich und ökologisch optimale Gestaltung des Projektes ein. Spätestens nach der Genehmigung braucht es dann einen finanzstarken Partner und während des Betriebes einen kompetenten Partner für die Betriebsführung und Vermarktung des Stromes. Diese Stärken werden am besten durch Energieversorgungsunternehmen (EVUs) eingebracht. Idealerweise spiegeln sich diese Stärken auch in der Beteiligung in den verschiedenen Projektphasen wider. Die Gemeinden und der Projektentwickler sind in den frühen Phasen stärker vertreten, die EVUs steigen spätestens bei der Genehmigung ein und sind während der Bau- und Betriebsphase stärker vertreten.
Der Projektentwickler steigt im Allgemeinen nach erfolgreicher Inbetriebnahme aus.

Damit sind in den verschiedenen Phasen eines Projektes auch die Risiken unterschiedlich verteilt. Die Entwicklung ist dann erfolgreich abgeschlossen, wenn am Ende des Prozesses eine Genehmigung erhalten und das Projekt finanziert werden kann. Das Risiko, dass der Entwicklungsaufwand zu keiner Genehmigung und Realisierung des Projektes führt, ist immer gegeben. Da die Gemeinden als zumeist finanzschwache Gebietskörperschaften ohne Unterstützung kaum in der Lage sind, die finanziellen Risiken für die Projektentwicklung auch nur anteilig zu übernehmen, verbleiben die Kosten in diesem risikobehafteten Stadium fast ausschließlich beim Projektentwickler. Aus diesem Grund kommt der genauen und kritischen Einschätzung der Genehmigungsfähigkeit des Projektes eine besondere Rolle zu. Die ursprüngliche Idee der Infra Project Development $\mathrm{GmbH}$, das Genehmigungsrisiko der Gemeinden durch eine Haftung des Landes abzudecken, ist bis heute nicht umsetzbar. Diese aus Sicht der Autoren sinnvolle Idee würde vorsehen, dass durch eine strenge Prüfung des Projektes durch eine Landesbehörde die Realisierungswahrscheinlichkeit beurteilt würde. Bei positiver Einschätzung des Projektes und seiner Genehmigungsfähigkeit sollte den Gemeinden aus öffentlichen Mitteln des Landes eine anteilige Finanzierung der Entwicklungskosten zur Verfügung gestellt werden. Bei erfolgreicher Realisierung des Projektes könnte dieser Betrag mit entsprechender Verzinsung refundiert werden. Damit ergäbe sich für das Land die Möglichkeit, Gemeinden und ihre langfristige Finanzkraft durch geringen Einsatz von Finanzmitteln zu fördern. Langfristig ergäbe sich sogar eine Verbesserung der Landesfinanzen, da die Erträge aus realisierten Kraftwerken die Finanzkraft der Gemeinden steigern und damit notwendige Zuschüsse aus den Landesmitteln verringern würden. Da diese Vorgehensweise seitens der Landesregierung nicht unterstützt wurde, musste der Projektentwickler bis zum Einstieg der EVUs das finanzielle Risiko alleine tragen.

Da Kraftwerksbauten erst nach Rückzahlung der Kredite höhere gesicherte Einnahmen erwirtschaften, ist es bei der Beteiligung an Wasserkraftprojekten für Gemeinden wichtig, dass 
zumindest kleinere Erträge bereits während der Kreditrückzahlung dem Gemeindebudget zufließen. Dies erleichtert es Gemeinderäten und Bürgermeistern, Entscheidungen zu treffen, deren positive Auswirkungen erst in viel späteren Legislaturperioden zum Tragen kommen. Damit schafft sich die Gemeinde die Möglichkeit, nicht nur den Ausbau erneuerbarer Energien im Interesse des Umweltschutzes zu forcieren, sondern auch zusätzliche Einnahmen zu erzielen. Eine Streckung der Rückzahlung des Kredits ermöglicht es den Gemeinden, kleinere Erträge auch in den ersten Jahren der Finanzierung zu sichern. Auch die Einnahmen während der Bauphase (Kommunalsteuer, Nächtigungen, Beteiligung lokaler Betriebe an der Errichtung) können nicht unwesentliche Beiträge zur Finanzierung des Eigenkapitalbedarfes der Gemeinden leisten.

Schließlich sei auch auf die zwangsläufig ungleiche Unternehmenskultur einer Gebietskörperschaft und eines privatwirtschaftlich geführten Unternehmens hingewiesen. Während Entscheidungen in Unternehmen nur von wenigen Personen und damit rasch getroffen werden können, ist der Entscheidungsprozess in den Gemeinden mit notwendiger umfassender Information aller Beteiligten ungleich komplexer und langsamer. Auch sind die Interessen Beider nicht in allen Punkten gleich gelagert. Deshalb ist eine Vertrauensbasis zwischen den handelnden Personen und Verständnis für die unterschiedlichen Gegebenheiten umso wichtiger, um mögliche Missverständnisse und daraus resultierendes Misstrauen zwischen den Partnern zu vermeiden und zeitgerecht zu gemeinsamen Entscheidungen zu kommen. Die Kombination eines rasch entscheidenden Unternehmens und einer im wahrsten Sinn des Wortes „,bedächtigen“ Vorgehensweise einer Gemeinde ist manchmal schwierig, bietet aber auch die Chance, zu besser durchdachten Entscheidungen zu kommen.

\subsection{Entwickeltes Gemeindebeteiligungsmodell}

Aus den vorher dargelegten Überlegungen ergibt sich das Gemeindebeteiligungsmodell der Infra $\mathrm{GmbH}$, das eine neue Möglichkeit der Zusammenarbeit zwischen Gemeinden und privatwirt- schaftlich geführten Unternehmen zur Umsetzung von Infrastrukturprojekten darstellt. Dabei werden die Stärken und Schwächen der Partner analysiert und daraus Strategien für eine erfolgversprechende Projektplanung entwickelt. Beispielsweise werden auch Vorgehensweisen entwickelt, die es ermöglichen, Gemeinden finanziell zu beteiligen, trotz mangelnder und gesetzlich begrenzter Möglichkeiten Risikokapital zu investieren. Für die EVUs bringt es den Vorteil, durch die Integration der Gemeinde eine erhöhte regionale Akzeptanz zu erreichen, wodurch Infrastrukturprojekte rascher umgesetzt werden können.

\subsubsection{Entwicklungsphase (von der ersten Idee bis zur Genehmigung)}

In der Entwicklungsphase übernimmt der Projektentwickler die Projektsteuerung und Planung. Die Gemeinden unterstützen das Projekt durch Einbringen der lokalen Projekterfordernisse bei der Gestaltung des Projekts, helfen bei der Beschaffung von Grundstücksrechten und spielen eine wesentliche Rolle in der Kommunikation mit allen Projektbeteiligten. Vor der Einreichung des Projekts zur behördlichen Genehmigung wird eine Projektgesellschaft mit Anteilen von etwa $50 \%$ für die Gemeinden und $50 \%$ für die Infra $\mathrm{GmbH}$ gegründet, was trotz ungleicher Verteilung des finanziellen Risikos dadurch begründet wird, dass beide Partner wesentlich zur Genehmigungsfähigkeit des Projekts beitragen.

In Abbildung 2 ist die Entwicklung von Eigenkapitalinvestitionen und des Projektwerts des Wasserkraftwerkes Stanzertal dargestellt. Der Wert des Projekts am Beginn ist gering; es handelt sich eigentlich nur um eine Idee mit einem Grobkonzept. Durch die beginnenden Tätigkeiten in den Bereichen Planung, Untersuchung und politische Umsetzung nehmen die Kosten zu, während der Wert des Projekts nur geringfügig steigt. Erst wenn die Chance auf eine Genehmigung und damit auf eine Realisierung zunimmt, beginnt eine relevante Wertsteigerung des Projekts. Eine derartige Einschätzung könnte beispielsweise durch die Beurteilung nach einem Kriterienkatalog erfolgen (Amt der Tiroler Landesregierung 2011), durch die Kommentare zu einem Konzept für eine Umweltverträglich- 
Abb. 2: Entwicklung von Eigenkapitalinvestitionen (EK) und Projektwert des WKW Stanzertal

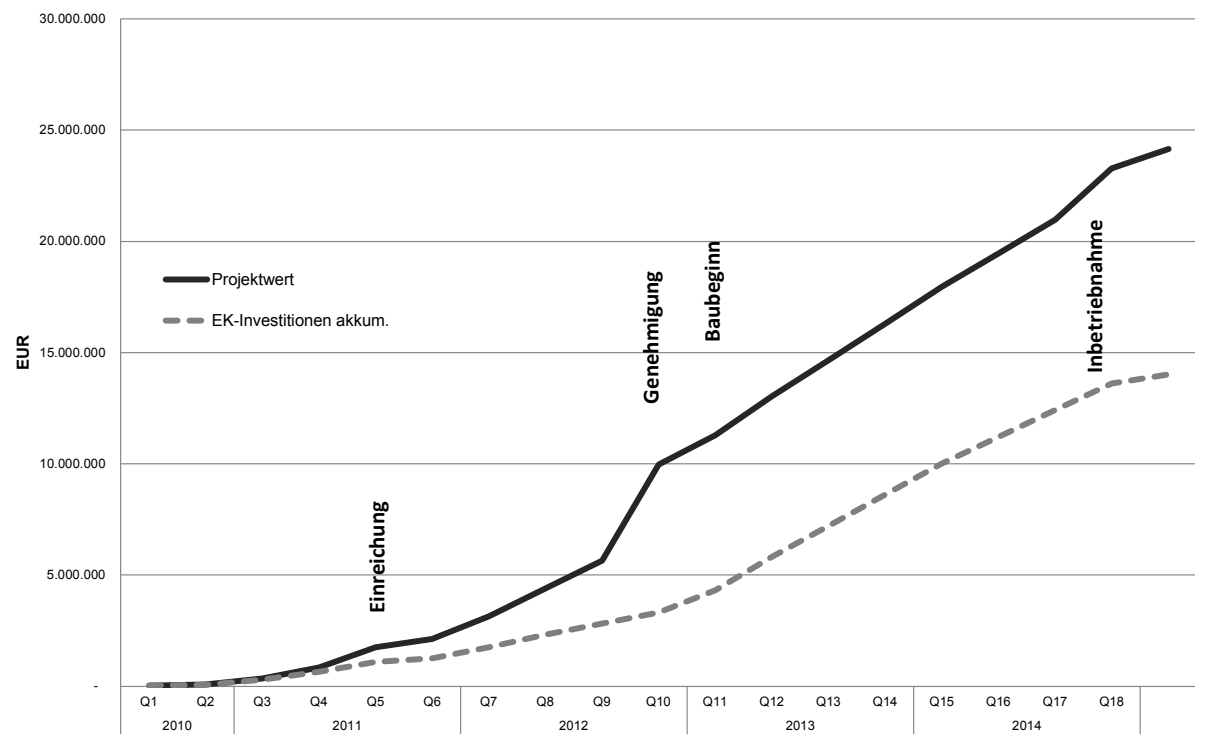

Quelle: Eigene Darstellung

keitserklärung (bei Umweltverträglichkeitsprüfungspflicht) und durch Rückmeldungen aus der Landespolitik. Mit Erhalt einer Genehmigung ist ein relevanter Projekt-Risikofaktor beseitigt und der Projektwert macht einen signifikanten Sprung nach oben.

\subsubsection{Bauphase (von der Genehmigung bis zur Inbetriebnahme)}

Zwar ist die Bauphase die finanziell aufwändigste Phase, allerdings sind die Realisierungschancen durch das Vorliegen der behördlichen Genehmigungen für das Projekt wesentlich erhöht. $\mathrm{Ab}$ diesem Zeitpunkt ist es für Energieversorgungsunternehmen (EVU) und Banken interessant, in das Projekt einzusteigen.

Die EVUs ersparen sich eigene risikobehaftete Planungen mit der Gefahr einer Nichtrealisierung und sind deshalb bereit, für den Einstieg in die Projektgesellschaft zu diesem Zeitpunkt einen entsprechenden finanziellen Beitrag zu leisten. Sie kaufen Anteile von den Gemeinden und von der Infra $\mathrm{GmbH}$, sodass in der Bauphase Gemeinden, EVUs und der Projektentwickler Partner in der Projektgesellschaft sind. Der Kaufpreis deckt nicht nur die aufgewendeten Vorkosten, sondern auch die Wertsteigerung durch die Genehmigung ab. Mit diesem Ertrag aus dem Verkauf einiger ihrer Anteile und einer üblichen Entschädigung für die Beeinträchtigungen im Tal während $\mathrm{Bau}$ und $\mathrm{Be}$ trieb des Projekts, können nun die Gemeinden zumindest einen wesentlichen Teil des anteiligen Eigenkapitals aus eigenen Mittel finanzieren. Den Rest müssen sie über einen Kredit aufnehmen, was bei rechtskräftiger Genehmigung und Ausfinanzierung des Projekts möglich ist.

Für die Banken ist ein genehmigtes Wasserkraftprojekt mit Aussicht auf sichere Erlöse nach Abschluss der Bauphase ein interessantes Projekt für eine Kreditvergabe. Ungefähr $70 \%$ der Investitionskosten können als Projektfinanzierung, d. h. ohne zusätzliche Sicherheiten durch die Gesellschafter, aus Bankkrediten aufgebracht werden und lediglich $30 \%$ müssen als Eigenkapital mit voller Haftung der Gesellschafter aufgebracht werden.

\subsubsection{Betriebsphase}

Nach Abschluss der Bauphase veräußert die Infra $\mathrm{GmbH}$ ihre Anteile vorzugsweise an die Gemeinden und/oder die Energieversorgungsunternehmen, sodass ab diesem Zeitpunkt die Gemeinden mit den EVUs partnerschaftlich als Betreiber des Kraftwerkes agieren. Die EVUs als wesentlicher Kompetenzträger für Betriebsführung und Vermarktung des Stroms und als finanzstarker Partner (um auch Jahre mit geringen Niederschläge oder Zeiten mit niedrigen Strompreisen zu überstehen) halten die Mehrheit der Anteile. Die Gemeinden profitieren von ihrer Beteiligung und sorgen damit nachhaltig für die regionale Akzeptanz des Projekts. 


\section{Gemeindebeteiligung am Beispiel Stanzertal}

\subsection{Projektstand}

Im Juli 2010 fanden die ersten Gespräche der Infra $\mathrm{GmbH}$ mit den Standortgemeinden bezüglich einer gemeinsamen Entwicklung des Projekts statt. Im August 2011 wurde das Projekt zur Genehmigung eingereicht und im Dezember 2012 wurde es genehmigt. Die verbindliche Finanzierungszusage lag Ende Februar 2013 vor. Da die Ausschreibungen parallel zum Genehmigungsverfahren durchgeführt wurden, konnte der Bau im März 2013 begonnen werden. Die Inbetriebnahme der ersten Turbine ist im 4. Quartal 2014 erfolgt, die Übernahme der Gesamtanlage im Juni 2015. Somit ergeben sich 5 Jahre von der ersten Idee bis zur Fertigstellung des Projekts. Diese rasche Verwirklichung war nur durch die gute und vertrauensvolle Zusammenarbeit aller Projektbeteiligten (Behörden, Standortgemeinden und betroffene Bürger, Grundeigentümer, Ausführende, Lieferanten, Energieversorgungsunternehmen und Entwickler) möglich und dokumentiert auch das gute Funktionieren der Gemeindebeteiligung.

\subsection{Beteiligungsprozess}

Knapp vor der Einreichung zur Genehmigung gründete die Infra $\mathrm{GmbH}$ die Projektgesellschaft WKW Stanzertal GmbH (,WKWSt") und hinterlegte notariell eine Abtretungsoption von 50\% der Anteile für die vier Stanzertaler Gemeinden. Ein sofortiger Eintritt der Gemeinden war nicht möglich, da die Finanzierung der Gemeindeanteile noch nicht gesichert war. Während des Genehmigungsprozesses wurde die Finanzierung der Gemeindebeteiligung in einer Rahmenvereinbarung zwischen später eintretenden EVUs (Elektrizitätswerke Reutte AG, Stadtwerke Imst und Energie- \& Wirtschaftsbetriebe der Gemeinde

Quelle: Eigene Darstellung
St. Anton [„EWA“]), den Stanzertaler Gemeinden (St. Anton, Pettneu, Flirsch und Strengen) und der Infra GmbH gesichert. Dabei wurde sichergestellt, dass die Standortgemeinden ihren gesamten Eigenkapitalbedarf bis zur Genehmigung und einen wesentlichen Teil ihres Eigenkaptalbedarfs während der Bauphase aus nach üblichen Regeln bemessenen Entschädigungen von Standortgemeinden für Kraftwerksbauten und Erlösen aus dem Verkauf von 25\% ihrer Anteile abdecken können.

Durch diese Vorgangsweise wurde gewährleistet, dass die Gemeinden in der Genehmigungsphase keine über das eingebrachte Stammkapital hinausgehenden Risiken eingehen mussten. In der Bauphase mussten sie zusätzlich nur für den fremdfinanzierten Teil ihrer Gesellschafterdarlehen haften, was von der Gemeindeaufsichtsbehörde nach einer Darstellung der wirtschaftlichen Gesamtsituation des Projekts ohne Probleme genehmigt wurde.

Nach Abschluss des Rahmenvertrags stand dem Eintritt der Gemeinden mit 50 \%-Anteilen in die WKWSt nichts mehr im Wege. Einige Wochen später waren auch die EVUs in die WKWSt eingetreten und etwa ein halbes Jahr später die Gemeinde Zams. Die Standortgemeinden und die Infra GmbH hatten beim Eintritt der EVU ihre Anteile entsprechend reduziert. Der Projektentwickler verkaufte seine restlichen Anteile nach Inbetriebnahme bevorzugt an die Partner, sodass sich am Ende die Gemeinden mit den EVUs im Verhältnis von $30-40 \%$ zu 70-60\% die Anteile an der WKWSt teilen konnten. Abbildung 3 zeigt

\section{Abb. 3: Entwicklung der Beteiligungsverhältnisse}




die Beteiligungsverhältnisse bei Gründung, während des Baus und nach Inbetriebnahme.

\subsection{Finanzierung}

Die Finanzierung des Kraftwerkes setzt sich wie folgt zusammen:

Stammkapital

Gesellschafterzuschuss

Gesellschafterdarlehen

240.000 Euro

OeMAG ${ }^{3}$ Förderung

750.000 Euro

12.275.000 Euro

4.600.000 Euro

Bankkredit

39.780.000 Euro

Gesamtinvestition (budgetiert) 57.645.000 Euro

Der Großteil des Eigenkapitals wurde als Gesellschafterdarlehen eingebracht, was bei der erwarteten Liquidität schon vor Erreichen der Gewinnzone Zinszahlungen an die Gesellschafter ermöglichte. Damit kamen alle Gesellschafter vom ersten vollen Betriebsjahr an in den Genuss von Zahlungen, was insbesondere für die Gemeinden interessant war, um das von ihnen aufgenommene Darlehen zurückzahlen und ihr Budget aufbessern zu können. Wann es zu zusätzlichen Ausschüttungen aus Gewinn der Gesellschaft kommt, hängt hauptsächlich von der Strompreisentwicklung ab.

Der Bankkredit wurde als reine Projektfinanzierung abgeschlossen, was bedeutet, dass die Gesellschafter für den Bankkredit nicht haften mussten. Die Banken verlangten lediglich eine betraglich limitierte Baukostenüberschreitungsund Schuldendienstreservegarantie, eine Garantie für den Ausfall der erst nach Inbetriebnahme endgültig fixierten OeMAG-Förderung sowie eine Strompreisgarantie für die ersten drei Betriebsjahre. Die Standortgemeinden wurden von diesen Garantien ausgenommen und die restlichen Gesellschafter verpflichteten sich, im Risikofall das notwendige Kapital als asymmetrisches Darlehen mit bevorzugten Konditionen zur Verfügung zu stellen. Um eine ausreichende Liquidität in den ersten Betriebsjahren zu gewährleisten, wurde ein Tilgungsplan des Bankkredites mit sehr geringen Tilgungen in den ersten Jahren vereinbart. Es ist geplant, nach einigen Betriebsjahren eine Refinanzierung vorzunehmen, da dann kein Bauzeit- und Baukostenrisiko mehr besteht und somit etwas günstigere Konditionen erreichbar sein müssten.

\subsection{Resümee}

In einer Zeit von 33 Monaten von der ersten Idee bis zum Spatenstich zu kommen, ist nur durch das gute und vertrauensvolle Zusammenwirken aller Beteiligten gelungen. Es wurde immer darauf geachtet, dass jeder seine Stärken einbringt und so die Schwächen der verschieden Partner nicht oder nur sehr eingeschränkt zum Tragen kamen. In den Gesellschafterversammlungen, bestehend aus Energieversorger, Gemeinden und dem Projektentwickler, wurden bis heute nur einstimmige Beschlüsse gefällt, was sehr nachdrücklich die öffentliche Akzeptanz des gemeinsamen Weges dokumentiert.

\section{Zusammenfassung und Ausblick}

Unsere Erfahrungen zeigen, dass durch die frühe Einbindung der Gemeinden in die Projektentwicklung und die Beteiligung an der Projektgesellschaft die NIMBY-Haltung überwunden werden kann. Voraussetzung dafür ist ein ökologisch verträgliches und wirtschaftliches Projekt. Schlüssel für das Erreichen der regionalen Akzeptanz ist der Aufbau eines Vertrauensverhältnisses unter den Projektpartnern. Dies gelingt durch Transparenz, ergebnisoffene Diskussionen mit den Partnern, Beteiligung an Entscheidungen und Erträgen sowie ein glaubwürdiges Bemühen, den gemeinsamen Projekterfolg über die Eigeninteressen zu stellen.

Nicht verschwiegen werden soll, dass bei dieser Vorgangsweise auch einige Herausforderungen zu bewältigen sind, die jedoch bei einer proaktiven Vorgehensweise lösbar zu sein scheinen:

- Risikoübernahme von Gemeinden: Gemeinden als Partner in der Projektgesellschaft müssen unternehmerisches Risiko übernehmen, was nur in eingeschränktem Maße möglich und zulässig ist. Hier wäre es wichtig, auch von öffentlicher Seite her diese Einschränkungen durch entsprechende Maßnahmen zu mindern. Damit läge die Last der Risikoübernahme nicht ausschließlich auf den Schultern der nicht öffentlichen Partner, was letztlich die Beteiligungs- und Ertragsmöglichkeiten der Gemeinden erhöhen würde. 
- Akzeptanz des Modells bei EVUs: EVUs haben manchmal eine verständliche Skepsis und Scheu, mit Gemeinden eine gemeinsame Projektgesellschaft zu gründen. Die unterschiedlichen Interessen und auch Entscheidungsfindungskulturen erschweren die Partnerschaft ebenso wie die nicht symmetrisch verteilten finanziellen Risiken. Andererseits sollten die Vorteile der regionalen Akzeptanz der Projekte während der Genehmigungsphase, dem Bau und dem Betrieb diese Erschwernisse auch für die EVUs bei weitem aufwiegen. Dazu muss v. a. durch gut funktionierende Beispiele und Modelle noch Überzeugungsarbeit geleistet werden.

- Langfristige Finanzierung: Gemeinden brauchen von Anfang an zumindest kleine Erträge aus dem Projekt. Dies erfordert einerseits relativ wirtschaftliche Projekte und andrerseits langfristige Finanzierungen. Wasserkraftwerke sind sehr langlebige Projekte ( $>60$ Jahre) und eine langfristige Finanzierung sollte daher kein Problem darstellen. Für die Banken ist allerdings eine Finanzierung über einen Zeitraum von mehr als 20 Jahren schwer. Hier müssen noch neue Modelle entwickelt werden, denn endfällige Kredite und frühzeitige Refinanzierungen sind nicht in jedem Fall gute Lösungen.

\section{Anmerkungen}

1) Heiliger Sankt Florian / Verschon' mein Haus / Zünd' and're an!

2) Not In My BackYard

3) Abwicklungsstelle für Ökostromstromförderungen in Österreich

\section{Literatur}

Amt der Tiroler Landesregierung, 2011: Wasserkraft in Tirol, Kriterienkatalog, Kriterien für die weitere Nutzung der Wasserkraft in Tirol, Rev. 3

Bliem, M.G., 2013: Der volkswirtschaftliche Nutzen von Großprojekten - Beispiele für Energie- und Verkehrsinfrastrukturprojekte, Vortrag im Rahmen der IBET am 31.10.2013, Innsbruck

e3 consult GmbH, 2013: Erlöspotential des Wasserkraftwerks Stanzertal durch eine optimierte Speicherbewirtschaftung. Unveröffentlichte Studie im Auftrag der Unicredit
Götz, W.; Krauter, S.; Schwenzer, A., 2011: Erfolgsfaktoren für die Akzeptanz von Erneuerbare-EnergieAnlagen. In: Energiewirtschaftliche Tagesfragen et 61/3 (2011), S. 47-49

Süßenbacher, W.; Knaus, K.; Kabinger, A., 2015: Der kurzfristige Stromhandel in Österreich. 9. Internationale Energiewirtschaftstagung an der TU Wien

\section{Kontakt}

Dipl.-Ing. Robert Weidner

Infra Project Development $\mathrm{GmbH}$

Framsweg 16, 6020 Innsbruck, Österreich

E-Mail: r.weidner@infra.at 